\title{
Dynamic Capacity Routing in Networks with MTSP
}

\author{
Ranjana Ponraj ${ }^{1 *}$, George Amalanathan ${ }^{2}$ \\ ${ }^{1}$ Hindustan University, Chennai, Tamil Nadu, India. \\ 2 Periyar Maniammai University, Thanjavur, Tamil Nadu, India. \\ * RanjanaPonraj author. Tel.: 09840358724; email: ranji.viji97@gmail.com \\ Manuscript submitted September 5, 2015; accepted April 21, 2016. \\ doi: 10.17706/ijcce.2016.5.6.465-472
}

\begin{abstract}
Network computer connections are growing at a faster rate. There is a needs advanced algorithms that helps in optimizing the network performance. Classical optimization based approaches have been applied since long time. But there is a need for more flexible and advanced algorithmic approaches to model the increasingly complex networks. Optimization is to find the best solution, either maximum or minimum out of all feasible solutions. In this paper, we propose an extension Travelling salesman Problem (TSP) in a systemic way by means of the modified version of the Multiple Traveling Salesman Problem (MTSP). The aim of this paper is used to solve the routing problem by considering two objectives; one is the minimization of the total time travelled by the salesman considering the capacity of the edges. After providing the offline version of the problem we propose and prove the online version of the problem using the Dynamic capacity Routing (DCR) algorithm. This work gives all feasible tools that can help to solve the problem and provide experimental insights into feasible network instances based on real life and simulated data.
\end{abstract}

Key words: Capacity, dynamic, graphs, routing, optimization.

\section{Introduction}

Computer science is the science of abstraction. Computer scientists must create abstraction of real world problems that can be represented and manipulated in a computer. The set of connections between items can be modeled as graph. A Graph is a set of points called vertices or nodes and connected by lined called as edges or arcs. Many real world problems can be modeled as a graph. This paper explores the concepts in graph theory and its application in computer science to demonstrate the utility of graph theory. This paper solves the routing problem using multiple travelling salesman (MTSP) approach. MTSP is the extension of a well known travelling salesman problem. Here instead of one sales man several sales men would visit the customer nodes, so that each customer nodes will be visited exactly once. If the salesman departs from multiple depot it is known as multiple depot multiple travelling salesman problem [1]. The Travelling Salesman problem (TSP) is a well known combinatorial optimization problem. There are variations in TSP problems to solve the routing and scheduling problems. This paper considers the TSP with capacity of the edges and routing takes place in an optimal manner. The other issue addressed is the problem of network capacity optimization, because of its importance in the field of information technology. The traffic managed is based on the capacity of the network. Mid size and large size organizations must control network traffic behavior to get the resources to perform optimally [2]. If the edge capacity is dealt it can also be used to optimize the network capacity for managing the traffic. For a fixed network and equipment, the capacity is 
determined by the number of packets received per unit time. Weighted graphs are used for re implementing the network by increasing the capacity of the arc in a graph which has arc cost and arc capacity [3]. Since there are many decision variables, the problem becomes a typical combinatorial optimization problem which cannot be solved using conventional algorithms. The running time of the algorithm is polynomial in input size. After providing a polynomial time algorithm for the offline version of the problem, we proved the optimally polynomial time online algorithm based on re optimization approach.

\section{Literature Review}

This section describes the different types of graph algorithms used to find the shortest path and the applications of MTSP to some real life problems.

\subsection{Applications of Graph Theory in the Field of Computer Science}

Graph data base use graph representation with nodes, edges and properties to represent and store data. The graph structure has a key role in designing database. In Software engineering, data flow diagrams are used where vertices represent transformation and edges represents the data flows. In computer hardware, graph theory concepts are used to model the limitations of the physical layer. In Data structures graph are used for the logical or mathematical model of a particular organization. Image processing techniques can be improved using a graph theoretic approach. In operating system graph is used for job scheduling and simultaneous job execution. The entire web us a graph where the item are documents and the references or links or connections [4].

\subsection{Graph Theory Routing Algorithms}

The major role of graph theory in computer applications is the development of graph algorithms. Routing problem is a domain with an infinite number of final solutions. One of the possible approaches to solve such a problem is using graph theory. The mathematical analysis methodology for a weighted undirected graph for solving a shortest path routing problem is applied. The problem is focused on searching the shortest path within a graph. This search coincides with the network routing problem domain. Klamper et al. proposed the routing problem is with circular graph using Routing Information Protocol finds the shortest path and all possible walks through the specified sub graph [5]. Routing function for a packet is equivalent to finding the shortest path in the graph associated to the network. To find the minimum number of hops for un weighted graphs and the shortest path for weighted graphs. For example, the Breath first search algorithm is used find the shortest path from the root to all possible destinations. This produces a spanning tree. The dijkstra's algorithm is used to find the shortest path between a source vertex and all other vertices in a graph. A fuzzy number is adopted to improve the classical dijkstra's algorithm in a transportation network to find the shortest path [6]. The spanning tree algorithms are used to avoid redundancy and loop in a network. The TSP can be modeled as a Graph Pyramid and solves TSP sequentially by moving from city to city using minimal spanning tree in a top down manner to obtain near optimal solution [7].

\subsection{The Multiple Travelling Salesman Problem (MTSP)}

Multiple travelling salesman problem is a generalization of the well known Travelling Salesman Problem (TSP) are applied while considering several Hamiltonian tours. The MTSP is a NP-hard and has much application in Transportation and Logistics. The Literature related to the MTSP and is practical applications have been reviewed by Bektas [8]. Shim et al. presented a mathematical formulation of the MTSP which considers the objective function as the minimization of the weighted sum of the global distance traveled by the travelling salesmen and the distance travelled by the sales man [9]. The different approaches used to 
solve TSP is exact and approximation method. Jozefowiez et al. proposed a met heuristic method to build an approximate description of the efficient solution set [10]. B'erub et al. developed an exact approach [11].

\section{Problem Definition and Notation}

In this paper, the network is modeled as a graph with the following constraints. Consider a graph $G=(V, E)$, where $V$ is the set of $n$ nodes and $E$ is the set of e edges. Associated with each edge $(i, j) \in E$ is the cost or distance or weight $w_{i j}$ and the capacity denoted as $c_{i j}$. We assume that the depot is node 1 and there are $m$ salesman at the depot. We define a binary variable $x_{i j}$ for each edge $(i, j) \in E ; x_{i j}$ takes the value 1 if edge $(i, j)$ is included in the tour and $x_{i j}$ takes the value 0 otherwise. We define an integer variable $U_{i}$ to denote position of node $i$ a tour, and we define a value $M$ to be the maximum number of nodes that can be visited by any salesman. We define a binary variable $y_{i}$ takes the value 1 if the nodes is in online mode and $y_{i}$ takes value 0 other wise.

Objective is to Minimize

$$
\sum(i, j) \in E w_{i j} x_{i j}
$$

Constraints

Exactly $m$ salesmen depart from node 1

$$
\sum j \in V(i, j) \in E x_{1, j}=m
$$

Exactly $m$ salesmen depart from node 1

$$
\sum j \in V(j, 1) \in E x_{i, 1}=m
$$

Exactly one tour enters each node

$$
\sum i \in V(i, j) \in E x_{i j}=1, \forall j \in V
$$

Exactly one tour exist in each node

$$
\sum j \in V(i, j) \in E x_{i j}=1, \forall i \in V
$$

\section{Solution Construction}

In this study, the network capacity optimization is solved as a asymmetrical MTSP problem by modeling the network as a graph. When dealing with the network capacity problem, the network management has to consider different types of capacities [12]. Here capacities are taken based on the edge or road capacity. The capacity of the edge is structure to schedule the maximum number of packets per unit time. Dealing with different capacities simultaneously is a complex problem. In the generalized form of MTSP the cities are represented as nodes. The task is then finding the shortest route, starting from the given node and visiting each node in the network exactly once before returning to the starting node. In this problem, we consider a source with 4 edges. The task of finding the shortest route based on the capacity of the edges. The study is restricted to the maximization of only 4 levels of capacity i.e. maximize the number of packets to deliver in the available capacity. The network is taken as a graph as shown in Fig. 1. 


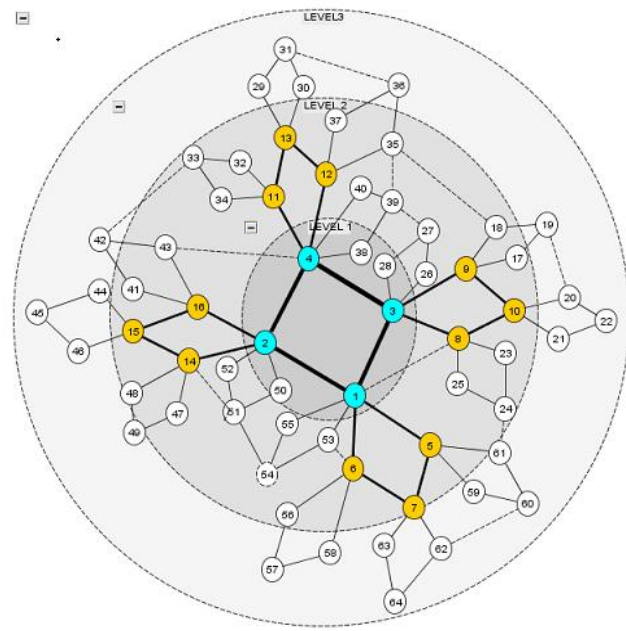

Fig. 1. Network with Capacity.

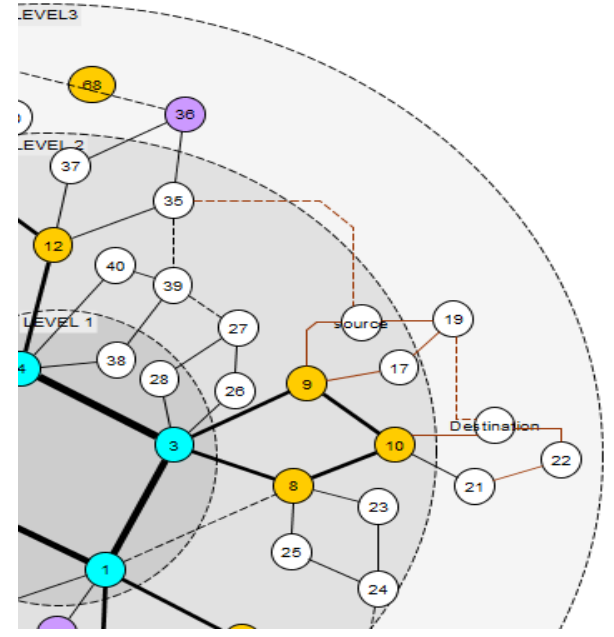

Fig. 2. Routing by DCRA.

\subsection{Network Capacity}

The complex network is modeled as a graph. The graph is divided in levels based on the capacity of the edges. In the above graph there are 3 levels. For each level the capacity of the edges differs. The edge with more capacity is placed in the leve 1 and with less that at level2 and so on. There were several algorithms to find the shortest path from source node to the destination node. But the PSPMS algorithm [13] uses multiple travelling salesman problem to find the route in parallel. In the network, if each salesman is assigned then the nodes which are nearer to each other will be served by different salesman, which is not efficient. To avoid this situation if a salesman find the nearby nodes he will visit that node even it is allotted to different salesman and he mark the node as visited so that the other salesman need not visit it again. In Fig. 2, node 20 can be reached easily by the salesman at 18 . But node 20 is allotted for different sale man and node 18 is allotted for other salesman, since the salesman at node 18 finds node 20 nearer to him he served that node 20. But for the salesman allotted to that node it will take a longer time. This issue is addressed and solved in the proposed Dynamic Capacity routing algorithm (DCRA).

\section{Algorithm 1:DCRA}

//Input: A Graph $(N, E, C)$ where a set $C$ is the capacity of all the edges. The starting node is taken as ' $s$ ' and the ending node is taken at ' $t$ ' in the Graph $G$

//Output: The shortest path between the node s and node $t$ with distance $d$

\section{1:Begin}

2:for each node ' $n$ ' in a graph $G$.

3: $\quad$ find the adjacent of $V$ with same capacity.

4: $\quad$ Add to Priority Queue()

5: $\quad$ Remove from Priority Queue().

6: $\quad$ If visited =false;

7: $\quad$ If mode $=$ on;

8: $\quad$ Mark visited as true

9: $\quad$ Assign name as $t$;

10: $\quad$ from source node $s$ to $t$

11: find the route using Dijkstras algorithm ;

12: $\quad$ Repeat till all nodes are visited;

13: If mode is off

14: calculate shortest route;

15: Repeat the above procedure for all levels

16:End; 
The proposed algorithm consists of a reaching the nearby node of same capacity by the same salesman who is nearer instead of making the other salesman who is allotted to that node to serve him. This algorithms also takes care of online or offline mode by assigning a variable called mode with on or off.

\subsection{Routing Process}

The sequence of steps for performing the algorithm is simulated using the YEd Graph tool. Fig. 3(a), Fig. 3(b) and Fig. 3(c). show the routing performance form source node to destination node in a graph. The source node every time performs one step Breath First Search (BFS) to find the adjacent nodes. It inserts the adjacent nodes onto the priority queue if they are in the same level i.e. the capacity of the edges are same and performs DCR algorithm to obtain the route. Fig. 4 shows the condition of not considered routes when both nodes are not at the same level.

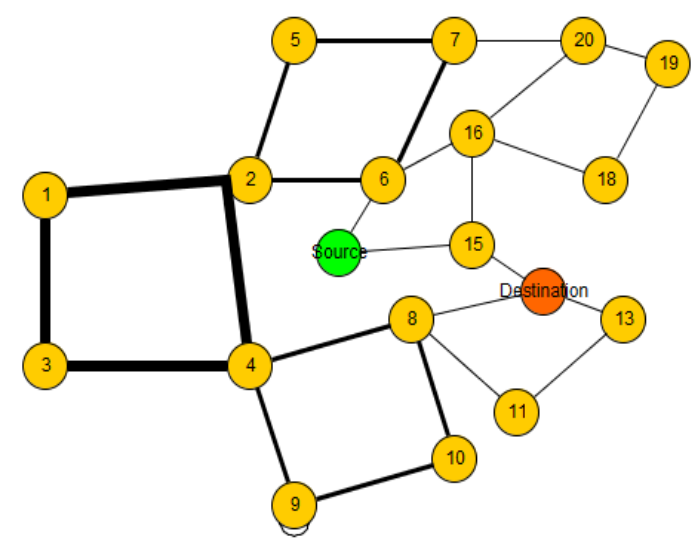

Fig. 3(a). Step 1 routing by DCRA.

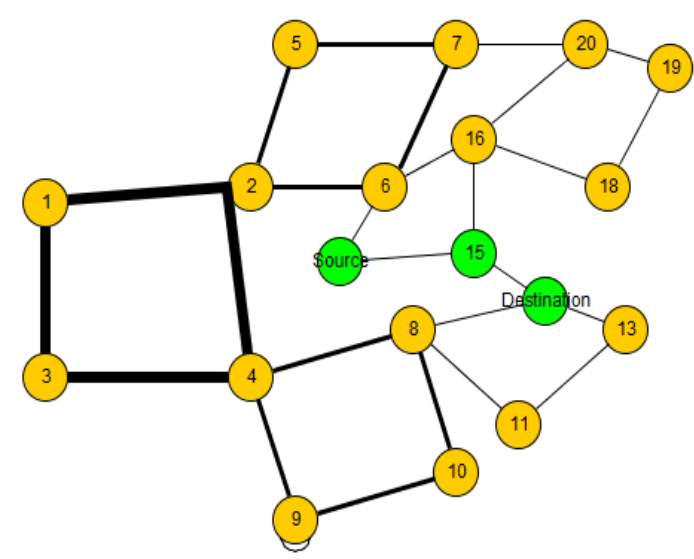

Fig. 3(c). Step 3 routing by DCRA.

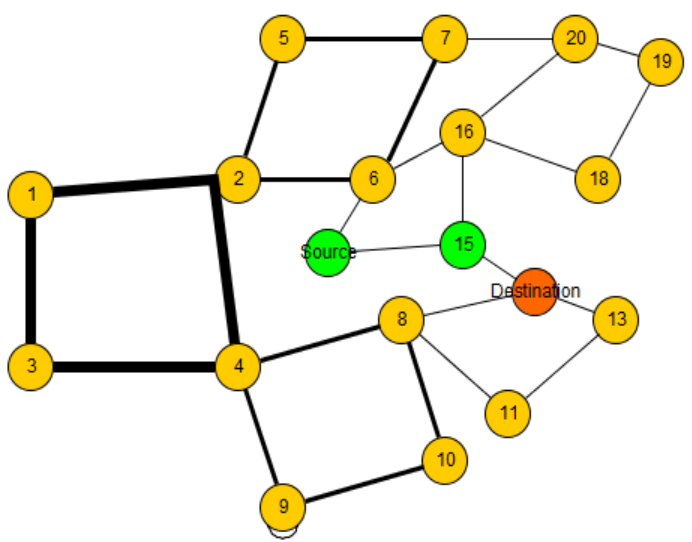

Fig. 3(b). Step 2 routing by DCRA.

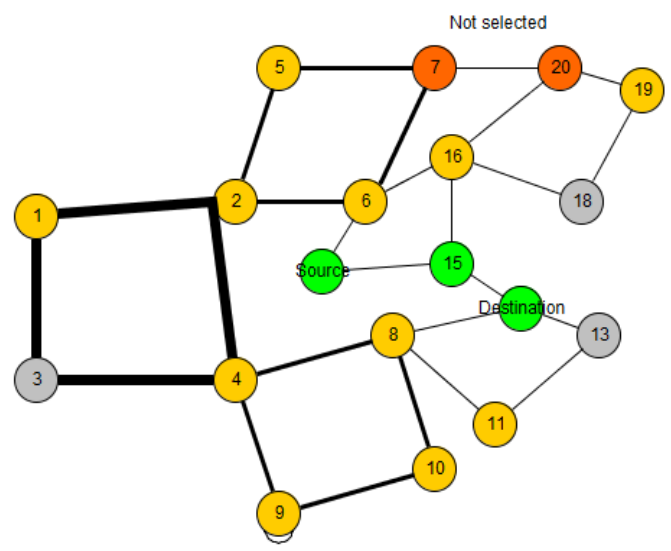

Fig. 4. Not selected route.

\section{Results and Discussions}

In this paper, we present some simulation results to compare the several routing algorithm for static and dynamic network. The experiment is performed for 1024 nodes. The graph is converted into data using Gephi and through simulation it finds Density, Diameter, path and degree. Then that data is taken and applied as a input to solve DCR algorithm. The algorithm is coded using Visual $\mathrm{C}++$ and executed. It calculates the time for each operation on the input data. Table 1 shows the input and output of the PSPMS algorithm and the proposed DCRA using Graph pad Prism 6. 
Table 1. Time Taken for PSPMS and DCRA Algorithms

\begin{tabular}{|l|l|l|l|}
\hline Nodes & Distance & PSPMS(Time) & DCRA(Time) \\
\hline 63 & 4.14 & 47 & 37 \\
\hline 72 & 4.15 & 54 & 43 \\
\hline 82 & 4.16 & 64 & 52 \\
\hline 47 & 3.60 & 56 & 45 \\
\hline 90 & 3.70 & 61 & 49 \\
\hline 61 & 6.70 & 35 & 28 \\
\hline 90 & 2.80 & 67 & 54 \\
\hline 35 & 2.10 & 28 & 36 \\
\hline 19 & 6.90 & 14 & 42 \\
\hline
\end{tabular}

From the output, we analyze the results of the previous PSPMS algorithm and DCRA algorithm for different capacity of edges. Table 1 Shows the Performance of DCRA Algorithm for the distance between the nodes and time taken for the distance. The level1 nodes are served only by one salesman. So, there will not be any difference in this level when compared to the other existing algorithms. Level 2 nodes are served by 4 salesmen sot there will a difference in time when it is treated using DCRA. Table 1 shows the data for level 2 of nodes.

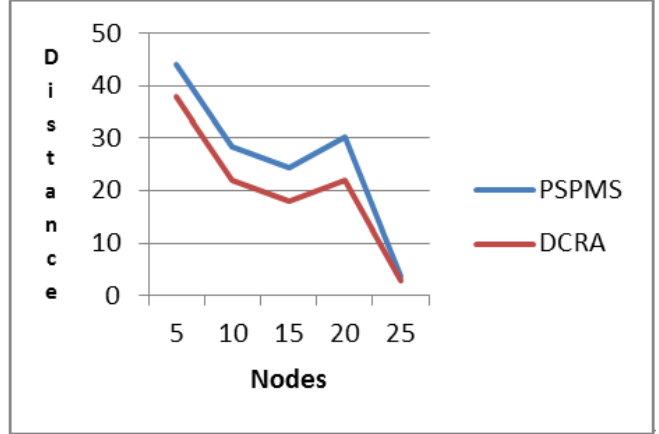

Fig. 5. DCRA for Level 2 nodes.

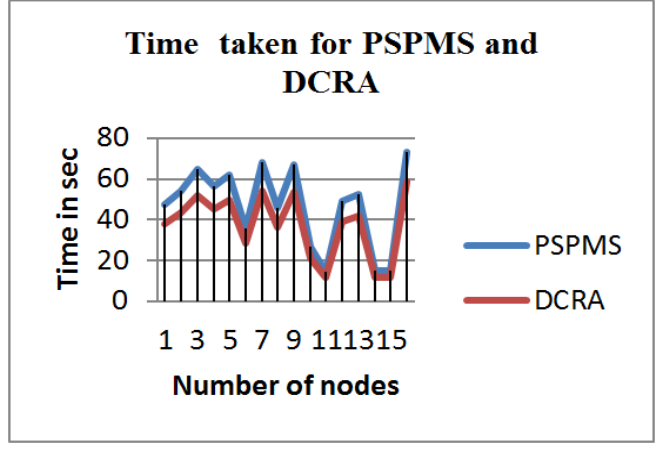

Fig. 6. Time and distance for PSPMS.

Fig. 5 shows the performance of DCRA for level1. At this level there will not be any change in the algorithm since it is served by one salesman. The time taken and distance covered for the nodes are plotted. There is an improvement in performance since it is served by 4 salesmen. Fig. 6 shows the time taken for 16 nodes of level 2 using PSPMS and DCRA algorithm. I this Level 2 nodes will be served by 4 salesmen also the salesman who is nearer will access the nodes by generating a first level of BFS. The nodes that are not participating can also be left since they will be in offline mode. Only nodes in online mode are taken into consideration.

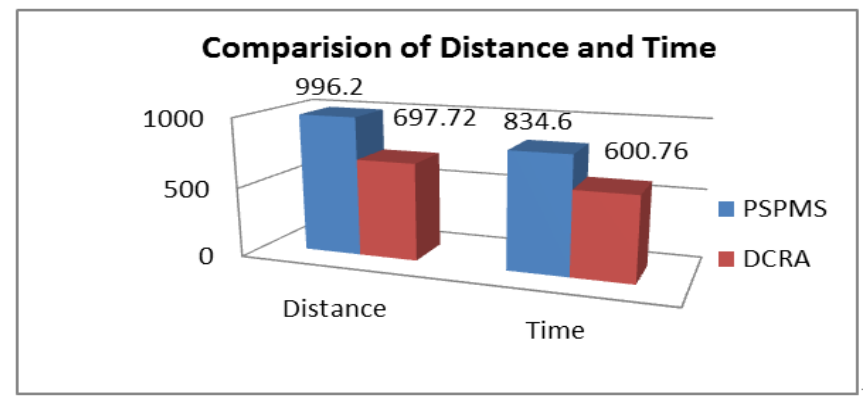

Fig. 7. Speed and time for PSPMS Vs. DCRA. 
Fig. 7 shows the comparison of the PSPMS and DCRA algorithm in terms of distance and time. The experimental results show that the performance of DCRA algorithm is able to perform the operation with less time compared to the PSPMS algorithm as shown in Fig. 7. The result of the experiments validate the formulation of the network capacity problem as an asymmetric MTSP.

\section{Conclusion}

The idea behind the problem was the search of flexible and advanced algorithmic approaches to model increasingly complex network configurations. We study the two-object one to minimize the time and another to address the dynamic issue in the network using graphs. The benefit of this method consists in the simplicity of the iteration formulae. The long time needed to reach the solution is motivated by the large number of elementary operation to do; thus, the performances are proportional to the number of nodes of the graph. Compared to the experimental results demonstrated the efficiency of the algorithm obtains answers very close to the optimal solution in very short time compared to the other existing approaches. Anyway, the time improvement we get by optimizing the data handling is evident and allows us to expect that additional code optimization can further improved the procedures performances.

\section{References}

[1] Ghafurian, S., \& Javadian, N. (2011). An ant colony algorithm for solving fixed destination multi-depot multiple travelling salesman problems. Applied Soft Computing, 11(1), 1256-1262.

[2] Kalyankar, N. V. (2009). Network traffic management. Journal of Computing, 1(1), 191-194.

[3] Demgensky, I., Noltemeier, H., \& Writh, H. C. (2004). Optimizing cost flows by edge cost and capacity upgrade. Journal of Discrete Algorithms, 2(4), 407-423.

[4] Shrinivas, S. G., Vertivel, S., \& Elango, N. M. (2010). Applications of graph theory in computer science an overview. International Journal of Engineering Science and Technology, 2(9), 4610-4621.

[5] Klamper, S., Mohorko, J., Cuce, S., \& Chowdhury, A. (2012) Graph's theory approach for searching the shortest routing path in RIP protocol: A case study. Przelad Elekrtotechnizny (Electrical Review). From http://pe.org.pl/articles/2012/8/58.pdf

[6] Deng, Y., Chen, Y.-X., Zhang, Y.-J., \& Mahadevan, S. (2012 March). Fuzzy Dijkstra algorithm for shortest path problem under certain environment. Applied Soft Computing, 12(3), 1231-1237.

[7] Haxhimasu, T., Kropatsch, W. G., Pizloz, Z., Ion, A., \& Lehrbaum, A. (2007) Approximating TSP solution by MST based graph pyramid approach. Graph-Based Representations in Pattern Recognition, 4538, 295-306.

[8] Bektas, T. (2006). The multiple traveling salesman problem: An overview of formulations and solution procedures. Omega, 34(3), 209-219.

[9] Shim, V. A., Tan, K. C., \& Tan, K. K. (2012). A hybrid estimation of distribution algorithm for solving multi-objective travelling salesman problem. Proceedings of IEEE Congress on Evolutionary Computation (pp. 1-8).

[10] Jozefowiez, N., Glover, F., \& Laguna, M. (2008). Multi-objective meta-heuristics for the traveling salesman problem with profits. Journal of Mathematical Modeling and Algorithms, 7(2), 177-195.

[11] B'erub'e, J., Gendreau, M., \& Potvin, J. (2009). An exact epsilon-constraint method for bi objective combinatorial optimization problems: Application to the traveling salesman problem with profits. European Journal of Operational Research, 194(1), 39-50.

[12] Gonasalves, T., \& Shiozaki, T. (2015). Solving capacity problem as a symmetric travelling salesman problem. International Journal of Artificial Intelligence and Application, 6(2), 53-65.

[13] Ranjana, P., \& George, A. (2014). Optimizing multiple travelling salesman problem considering the road 
capacity. Journal of Computer Science, 10(4), 680-688.

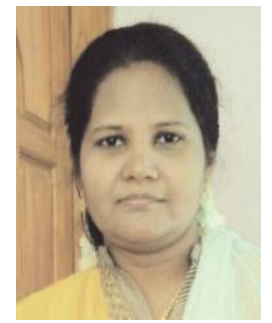

P. Ranjana received her master degree from Anna University, Chennai, India. She jointed Hindustan Group of Institutions in 1999. Now she is doing doctoral program in Hindustan University, Chennai, India. She has fifteen years of teaching experience in engineering colleges both undergraduate and postgraduate level. Her area of interest is computer networks, graph theory, combinatorial algorithms and artificial intelligence.

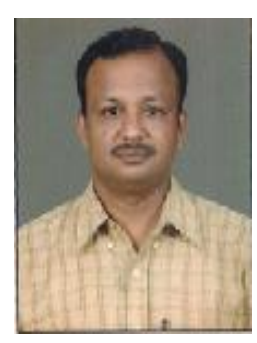

A. George received his PhD degree from IIT Madras. He joined in Hindustan Group of Institutions, Chennai in 2001. He is working as a professor in Periyar Maniammai University. He has more than 20 years of teaching experience in colleges both undergraduate and post graduate level. His research interests are applications of graph theory in mobile Ad Hoc networks, fuzzy theory and combinatorial algorithms. 